\title{
Incidence and Predictors of Flares in the Postpartum Year Among Women With Inflammatory Bowel Disease
}

\author{
Amy Yu, MD, ${ }^{*, \oplus}$ Sonia Friedman, $M D^{+}{ }^{+}$and Ashwin N. Ananthakrishnan, $M D, M P H^{*, \neq}$
}

Background: The postpartum period is marked by physiological and psychological stresses that may impact activity in inflammatory bowel disease. The predictors and outcomes of disease activity during this period have not been well characterized.

Methods: We performed a retrospective review of inflammatory bowel disease patients who underwent successful pregnancy and live birth at 2 referral institutions. Data on patient and disease factors including disease activity before and during pregnancy were abstracted from the medical records. We noted whether therapy was dose-reduced or stopped during pregnancy at each trimester and after delivery. Multivariable logistic regression of independent predictors of postpartum flare was performed, adjusting for relevant covariates.

Results: We identified a total of 206 eligible women (mean age, 33.2 years). Of these, 97 (47\%) had a diagnosis of Crohn's disease, whereas the remainder had ulcerative colitis. Nearly half the women delivered vaginally (53\%), and the rest delivered by Caesarean section (47\%). In the entire cohort, 65 (31.6\%) experienced a postpartum flare within the year after delivery. In multivariable analysis, development of a postpartum flare was predicted by disease activity during the third trimester (odds ratio [OR], 6.27; 95\% confidence interval [CI], 2.81-17.27), therapy de-escalation during pregnancy $(\mathrm{OR}, 3.00 ; 95 \% \mathrm{CI}, 1.03-8.68)$, and therapy de-escalation after pregnancy (OR, 4.43; 95\% CI, 1.55-12.65). Postpartum disease flare was not related to disease type, duration of disease, or mode of childbirth.

Conclusions: One-third of women with inflammatory bowel disease may experience disease flare during the postpartum year. Continued optimization of therapy before, during, and after pregnancy is essential to prevent this morbidity.

Key Words: Crohn's disease, ulcerative colitis, pregnancy, postpartum, relapse

\section{INTRODUCTION}

Crohn's disease (CD) and ulcerative colitis (UC) are chronic inflammatory diseases that frequently affect women in their reproductive years, with half of $\mathrm{CD}$ and UC patients diagnosed before age 35 years. ${ }^{1-3}$ As a result, the impact of inflammatory bowel disease (IBD) on pregnancy outcomes is an important consideration for both patients and providers. ${ }^{46}$ Many studies have investigated the influence of IBD

Received for publications October 8, 2019; Editorial Decision December 2, 2019.

From the *Department of Medicine, Massachusetts General Hospital, Boston, Massachusetts, USA; 'Division of Gastroenterology, Brigham and Women's Hospital, Boston, Massachusetts, USA; ${ }^{\ddagger}$ Division of Gastroenterology, Massachusetts General Hospital, Boston, Massachusetts, USA

Supported by: Ananthakrishnan is supported in part by grants from the National Institutes of Health (R03 DK112909), the Crohn's and Colitis Foundation, and the Chleck Family Foundation.

Conflicts of interest: Ananthakrishnan has served on scientific advisory boards for Abbvie, Takeda, Gilead, and Merck and has received research support from Pfizer. The other authors have no conflicts to declare.

Author contributions: Yu, Ananthakrishnan - study design, statistical analysis, and drafting of the manuscript. Yu - data extraction. Ananthakrishnan, Yu, Friedman - critical revision and approval of the final manuscript. Ananthakrishnan study supervision. All authors approved the final manuscript.

Address correspondence to: Ashwin N. Ananthakrishnan, MD, MPH, Division of Gastroenterology, Massachusetts General Hospital, 165 Cambridge Street, 9th Floor, Boston, MA 02114 (aananthakrishnan@mgh.harvard.edu).

(C) 2020 Crohn's \& Colitis Foundation. Published by Oxford University Press. All rights reserved. For permissions, please e-mail: journals.permissions@oup.com.

doi: 10.1093/ibd/izz313

Published online 2 January 2020 on fertility, delivery, and birth outcomes, noting that women with IBD may experience a higher rate of pregnancy-related complications and adverse pregnancy outcomes than the general population..$^{7-11}$ In particular, active disease during the time of conception increases the incidence of preterm delivery and fetal loss, whereas quiescent disease appears to have minimal effects on the course of pregnancy compared with non-IBD controls. ${ }^{9-12}$

In addition to the impact of IBD on pregnancy, the effect of pregnancy itself on the course of IBD both before and after delivery is an essential consideration for patients undergoing obstetric counseling. Despite growing literature on disease behavior during pregnancy, factors that impact the IBD course during the postpartum period have not been well defined. Many prior studies of IBD and pregnancy discount the immediate postpartum period. However, there are many factors in this period that may uniquely affect disease course. The postpartum period is associated with sharp decreases in both estrogen and progesterone. Breastfeeding further impacts hormonal levels during this period. In addition, the postpartum period may be characterized by increased levels of stress and sleep disruption associated with the care of a neonate, along with alterations in mood. ${ }^{13-15}$ The few studies that have examined postpartum factors have investigated medication nonadherence, resumption of smoking, and breastfeeding. ${ }^{15-17}$ Limitations of these prior studies include small sample sizes, inherent recall bias in questionnaire data, and short follow-up 
time after pregnancy. Furthermore, many represent findings before the introduction of biologics (and thus on drugs with shorter half-lives) for the treatment of IBD and consequently do not reflect contemporary treatment practices regarding cessation or continuation of therapy. ${ }^{15,16,18,19}$

Thus, we conducted a retrospective study to (a) identify the rate of postpartum relapse in large cohort of women with IBD and (b) determine the pre-, intra-, and postpregnancy predictors of IBD relapse in the postpartum year, during an era of frequent biologic use.

\section{METHODS}

\section{Study Population}

This study was a retrospective cohort analysis of women with a diagnosis of $\mathrm{UC}$ or $\mathrm{CD}$ who had at least 1 pregnancy and live birth at 2 major academic referral hospitals, Massachusetts General Hospital (MGH) and Brigham and Women's Hospital (BWH). These 2 hospitals together serve an area of $>3$ million patients in the Greater Boston metropolitan area. Eligible patients were identified using the Partners Research Patient Data Registry (RPDR), a comprehensive clinical data query system and repository of all inpatient and outpatient patient information including medications, laboratory tests, radiology, endoscopy, and surgical/obstetric procedures..$^{20}$ The use of this data repository to identify patients has been previously described in publications from our center. ${ }^{21,22}$ Approval from the Institutional Review Board at Partners Healthcare was obtained to evaluate the data.

For this study, we identified patients with a confirmed diagnosis of IBD using International Classification of Diseases, 9th and 10th Edition, Clinical Modification codes for UC (ICD-9-CM 556.x, ICD-10-CM K51.xx) or CD (ICD-9-CM 555.x, ICD-10-CM K50.xx). Of those patients, we concurrently identified those who also had at least 1 pregnancy and live birth at our institution between 2015 and 2018 using the diagnosis codes for vaginal or cesarian delivery (ICD-9-CM 650, 660669, ICD-9-CM 669.7, 74.0-74.9, ICD-10-CM Z37.0, O80-82). A chart review of the electronic medical record was performed to confirm the diagnosis of IBD, along with the pregnancy and delivery method. Only cases with IBD diagnosed before pregnancy or within a year after pregnancy were included in the analysis.

\section{Definition of Covariates}

We abstracted covariates including age of IBD diagnosis, ethnicity, IBD type, age of delivery, delivery type, pregnancy number, smoking history, and breastfeeding. Patients were classified using Montreal disease classification; CD patients were categorized based on location (ileal, colonic, or ileocolonic) and behavior (inflammatory, stricturing, or penetrating), whereas UC patients were classified based on disease extent (proctitis, left-sided, or extensive [pancolitis]). Crohn's disease patients were assigned the more severe phenotype if they had evolving disease behavior. A history of prior surgical procedure for IBD was documented. Active luminal disease immediately before pregnancy and during the 3 trimesters of pregnancy was noted, with disease activity during each trimester treated independently of each other. Active disease was ascertained based on documented history in gastroenterology provider notes (diarrhea, abdominal pain, rectal bleeding), endoscopy (presence of erythema or ulcerations), or radiology (wall thickening, increased vascularity, and mucosal enhancement).

The use of medical therapies including systemic steroids (oral or intravenous), immunomodulators (azathioprine, 6-mercaptopurine [6-MP], methotrexate), aminosalicylates (5-ASA; mesalamine, sulfasalazine, balsalazide), and biologics (infliximab, adalimumab, certolizumab, golimumab, natalizumab, vedolizumab, and ustekinumab) was defined before pregnancy, during pregnancy, and after pregnancy. Stopping 1 or more medical therapies or decreasing the dose or frequency of 1 or more therapies during pregnancy or after pregnancy was defined as therapy de-escalation and noted based on the electronic prescription function and documented gastroenterology notes. Smoking history was classified as never/ever smoked. Breastfeeding after delivery was ascertained based on lactation and postpartum notes.

\section{Outcomes}

Our primary study outcome was a postpartum IBD flare in the 12 months after delivery. A postpartum flare was defined as at least 1 of the following: (i) therapy escalation including new initiation of a medication or increasing the dose and/or frequency of a prior medication; (ii) IBD-related hospitalizations, defined as hospitalizations where IBD was determined to be primary diagnosis on discharge; or (iii) IBD-related surgery. A postpartum flare was documented based on the above criteria regardless of whether the patient had active disease before or during pregnancy. We repeated our analysis among patients with de novo postpartum flare, defined as absence of disease activity in the third trimester but presence of active disease postpartum. Furthermore, we identified patients in our cohort who had a new diagnosis of IBD during the pregnancy or postpartum year. A new diagnosis during the postpartum year was defined as a postpartum flare.

\section{Statistical Analysis}

All data were analyzed using Stata 15.1 (Stata Corp, College Station, TX, USA). Continuous variables were summarized with means and standard deviations, whereas categorical variables were described using proportions. The $t$ test was used to compare continuous variables, whereas the $\chi^{2}$ test was used to compare categorical variables, with use of the Fisher exact test when appropriate. Univariate logistic regression was 
performed to identify predictors of our primary outcome, a postpartum flare. Variables significant at $P<0.05$ in this analysis were carried forward into a multivariable regression model where a 2 -sided $P$ value $<0.05$ indicated independent statistical significance.

\section{RESULTS}

We identified a total of 206 patients with an established diagnosis of $\mathrm{CD}$ or $\mathrm{UC}$ who had at least 1 successful pregnancy and delivery at our institutions between 2015 and 2018. Of these patients, $97(47 \%)$ had a diagnosis of $\mathrm{CD}$, whereas 109 $(53 \%)$ had a diagnosis of UC. The mean age $( \pm S D)$ of IBD diagnosis was $22.8 \pm 7.1$ years, with mean duration of disease of $10.4 \pm 6.8$ years. The mean age of delivery was $33.2 \pm 4.9$ years, with an average pregnancy number of $1.8 \pm 0.9$. A total of 109 women $(53 \%)$ underwent vaginal delivery, whereas $97(47 \%)$ delivered via C-section. Over three-quarters of the cohort $(\mathrm{n}=180,87.4 \%)$ breastfed after delivery, and $29(14.1 \%)$ patients reported a smoking history in their lifetime. The group with active disease was comprised of 5 women $(2.4 \%)$ at the time of conception, $9(4.4 \%)$ during the first trimester of pregnancy, $16(7.8 \%)$ during the second trimester, and $34(16.5 \%)$ women during the third trimester. On follow-up, a total of 65 women $(31.6 \%)$ experienced a postpartum flare within the year after delivery. Among patients who experienced postpartum flares, $10(15.4 \%)$ were hospitalized with IBD as the primary diagnosis, 5 (7.7\%) underwent IBD-related surgery, and 63 (96.9\%) experienced disease activity leading to therapy escalation. Of these, 26 women (40\%) had CD, whereas $39(60 \%)$ had UC. Forty patients (19.4\%) experienced a de novo postpartum flare, and $12(3 \%)$ were newly diagnosed with IBD during pregnancy or in the postpartum year.

Table 1 demonstrates a univariate comparison between patients who experienced a postpartum flare vs those who did not. Women who experienced a postpartum flare were slightly younger than those who did not ( 31.7 vs 33.9 years; $P=0.002$ ) but were similar in terms of ethnicity and IBD type. There was no significant difference in postpartum flares depending on delivery type (vaginal vs cesarean) or pregnancy number between the 2 groups. Among the patients with $\mathrm{CD}$, disease location did not correlate with postpartum flares; however, women with more severe disease behavior (penetrating $>$ stricturing $>$ inflammatory) were more likely to experience a postpartum flare. In particular, $38.8 \%$ of patients with $\mathrm{CD}$ who experienced a postpartum flare had penetrating disease, compared with $7.6 \%$ of patients who did not experience a postpartum flare $(P<0.05)$. Among patients with $\mathrm{UC}$, there was no correlation with Montreal disease extent and postpartum flare. There was no difference in breastfeeding or lifetime smoking between women who experienced a postpartum flare and those who did not.

Patients who had active disease before pregnancy were more likely to experience a postpartum flare than to stay quiescent during the postpartum year $(7.7 \%$ vs $0 \% ; P=0.003)$. Similarly, active disease during each trimester of pregnancy was associated with having a postpartum flare (first trimester: $10.8 \%$ vs $1.4 \% ; P=0.005$; second trimester: $20 \%$ vs $2.1 \% ; P<0.001$; third trimester: $38.5 \%$ vs $6.4 \% ; P<0.001)$. There was no difference in medical therapy used during pregnancy across both groups, although there was a trend toward higher-frequency biologic use in patients who experienced a postpartum flare vs patients who did not ( $40 \%$ vs $26.2 \% ; P=0.05)$. Among patients who flared postpartum, $40 \%$ were on biologics, $25 \%$ were on 5 -ASA, $6 \%$ were on immunomodulators, and $5 \%$ were on systemic steroids during pregnancy. Patients who stopped (12.3\% vs $4.3 \% ; P=0.04)$ or decreased their medical therapy during pregnancy $(9.2 \%$ vs $1.4 \% ; P=0.01)$ (Fig. 1$)$ or stopped $(18.5 \%$ vs $2.1 \% ; P<0.001)$ or decreased their medical therapy after delivery $(9.2 \%$ vs $2.8 \% ; P=0.05)$ were more likely to experience a postpartum flare.

In a multivariable analysis summarized in Table 2, independent predictors of a postpartum flare were age (odds ratio [OR], 0.92; 95\% confidence interval [CI], 0.85-0.99), active disease in the third trimester (OR, 6.27; 95\% CI, 2.81-17.27), therapy de-escalation during pregnancy (OR, 3.00; 95\% CI, 1.03-8.68), and therapy de-escalation after pregnancy (OR, 4.43; 95\% CI, 1.55-12.65). Among patients with quiescent disease during the third trimester, therapy de-escalation after pregnancy (OR, 4.32; 95\% CI, 1.41-13.22) was an independent predictor of de novo postpartum flare.

In a subgroup analysis among the 63 patients on biologics during the time of conception, $26(41 \%)$ experienced a postpartum flare (Table 3). The presence of active disease during pregnancy, regardless of which trimester, and stopping or decreasing the biologic dose and/or frequency postdelivery ( $30.8 \%$ vs $8.1 \% ; P=0.04$ ) were predictors of flare among those taking biologics. There was no difference in postpartum flares based on whether women stopped their biologics during the first trimester $(15.4 \%$ vs $8.1 \% ; P=0.37)$ or third trimester of pregnancy $(50.0 \%$ vs $40.5 \% ; P=0.46)$.

Among our cohort, 6 patients received a new diagnosis of IBD during pregnancy, and 6 patients received a new diagnosis of IBD during the postpartum year. All 12 of these women were diagnosed with UC, whereas no patients were diagnosed with $\mathrm{CD}$ during pregnancy or during the postpartum year. Of the 6 diagnosed during pregnancy, $5(83 \%)$ experienced a continued flare or new flare during the postpartum year. These women with new IBD diagnoses tended to be younger at the age of delivery (age 30.1 vs 33.4 years; $P=0.02$ ) but older at the age of IBD diagnosis (age 30.3 vs 22.2 years; $P<0.01$ ) compared with women with prior IBD diagnoses.

\section{DISCUSSION}

As IBD often affects women of childbearing age, the examination of IBD outcomes during pregnancy is an important area to be studied. ${ }^{7-11}$ However, factors that impact the IBD 
TABLE 1. Characteristics of Women With Inflammatory Bowel Disease, Stratified by Postpartum Relapse Status

\begin{tabular}{|c|c|c|c|}
\hline Characteristic & Postpartum Flare $(\mathrm{n}=65)$, No. $(\%)$ & No Postpartum Flare $(\mathrm{n}=141)$, No. $(\%)$ & $P$ \\
\hline Age at delivery, mean (SD), y & $31.7(4.9)$ & $33.9(4.8)$ & 0.002 \\
\hline Delivery type & & & 0.19 \\
\hline Vaginal & $30(46.2)$ & $79(56.0)$ & \\
\hline Cesarean & $35(53.8)$ & $62(44.0)$ & \\
\hline Pregnancy number, mean (SD) & $1.7(0.9)$ & $1.8(0.9)$ & 0.85 \\
\hline Ethnicity & & & 0.08 \\
\hline White & $54(83.1)$ & $129(91.5)$ & \\
\hline Nonwhite & $11(16.9)$ & $12(8.5)$ & \\
\hline Age at IBD diagnosis, mean (SD), y & $22.7(6.7)$ & $22.8(7.3)$ & 0.93 \\
\hline Duration of disease, mean (SD), y & $9.3(6.7)$ & $11.0(6.8)$ & 0.11 \\
\hline Prior IBD surgery & $13(20.0)$ & $23(16.3)$ & 0.52 \\
\hline IBD type & & & 0.17 \\
\hline $\mathrm{CD}$ & $26(40.0)$ & $71(50.3)$ & \\
\hline $\mathrm{UC}$ & $39(60.0)$ & $70(49.7)$ & \\
\hline CD Montreal classification & $\mathrm{n}=26$ & $\mathrm{n}=58$ & 0.28 \\
\hline Ileal & $3(11.5)$ & $15(25.9)$ & \\
\hline Colonic & $3(11.5)$ & $8(13.8)$ & \\
\hline Ileocolonic & $20(77.0)$ & $35(60.3)$ & \\
\hline CD behavior & $\mathrm{n}=26$ & $\mathrm{n}=66$ & $<0.001$ \\
\hline Inflammatory & $9(34.6)$ & $52(78.8)$ & \\
\hline Stricturing & $9(34.6)$ & $9(13.6)$ & \\
\hline Penetrating & $8(38.8)$ & $5(7.6)$ & \\
\hline UC Montreal & $\mathrm{n}=38$ & $\mathrm{n}=67$ & 0.72 \\
\hline Proctitis & $11(29.0)$ & $15(22.4)$ & \\
\hline Left-sided & $11(29.0)$ & $23(34.3)$ & \\
\hline Extensive (pancolitis) & $16(42.0)$ & $29(43.3)$ & \\
\hline Active disease at time of conception & $5(7.7)$ & $0(0.0)$ & 0.003 \\
\hline \multicolumn{4}{|l|}{ Active disease by trimester } \\
\hline First & $7(10.8)$ & $2(1.4)$ & 0.005 \\
\hline Second & $13(20.0)$ & $3(2.1)$ & $<0.001$ \\
\hline Third & $25(38.5)$ & $9(6.4)$ & $<0.001$ \\
\hline \multicolumn{4}{|l|}{ Therapy during pregnancy } \\
\hline Systemic steroids & $3(4.6)$ & $1(0.7)$ & 0.09 \\
\hline Immunomodulators & $4(6.2)$ & $17(12.1)$ & 0.19 \\
\hline 5-ASA & $16(24.6)$ & $38(27.0)$ & 0.72 \\
\hline Biologics & $26(40.0)$ & $37(26.2)$ & 0.05 \\
\hline Biologic held 1st trimester & $4(15.4)$ & $3(8.1)$ & 0.37 \\
\hline Biologic held 3rd trimester & $13(50.0)$ & $15(40.5)$ & 0.46 \\
\hline Timing of last biologic, mean (SD), wk before delivery & $10.5(13.7)$ & $8.4(10.1)$ & 0.49 \\
\hline Therapy de-escalation during pregnancy & $14(21.5)$ & $8(5.7)$ & 0.001 \\
\hline Stopped therapy & $8(12.3)$ & $6(4.3)$ & 0.04 \\
\hline Decreased therapy & $6(9.2)$ & $2(1.4)$ & 0.01 \\
\hline Therapy de-escalation after delivery & $18(27.7)$ & $7(5.0)$ & $<0.001$ \\
\hline Stopped therapy & $12(18.5)$ & $3(2.1)$ & $<0.001$ \\
\hline Decreased therapy & $6(9.2)$ & $4(2.8)$ & 0.05 \\
\hline Breastfeeding & $55(84.6)$ & $125(88.7)$ & 0.42 \\
\hline Smoking (ever in lifetime) & $10(15.4)$ & $19(13.5)$ & 0.83 \\
\hline
\end{tabular}


course during the postpartum period have yet to be well defined. Our retrospective cohort analysis of 2 large tertiary referral centers sought to examine the incidence and predictors of IBD flare during the postpartum year. Among our cohort of 206 patients, a total of 65 women (31.6\%) experienced a postpartum flare within the year after delivery. Our results indicate that active disease and therapy de-escalation during pregnancy or postpartum were associated with disease flares during the postpartum year.

The first important finding from our study is the quantification of risk of IBD relapse after delivery, which, in our study, comprised nearly one-third of the cohort. This frequency is comparable to the limited literature on this topic, though most prior work examined outcomes primarily in the 6 months after delivery. A European cohort study from 2003 to 2006 reported the cumulative probability of retaining remission within the 6 months postpartum to be $71 \%$ for $\mathrm{CD}$ and $60 \%$ for UC. ${ }^{19}$ Studies using questionnaire data have noted self-reported disease relapse ranging from $14 \%$ to $29 \%$ during the 6 months to 1 year after delivery, though these studies are limited by inherent recall bias. ${ }^{16,17}$ These findings, together with ours, highlight that there remains a moderate risk of relapse of IBD in the

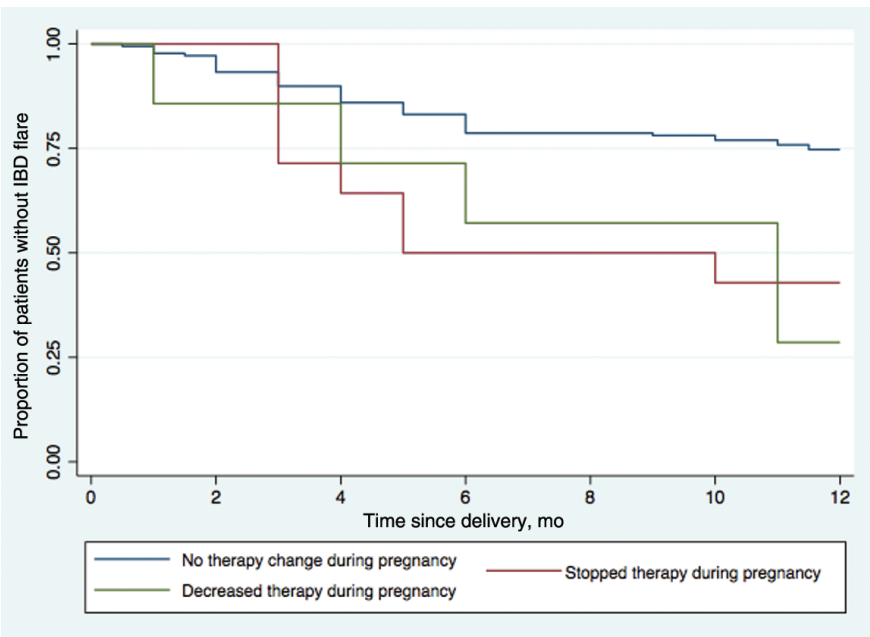

FIGURE 1. Risk of relapse in women with inflammatory bowel disease in the postpartum year, stratified by therapy changes during pregnancy. postpartum year that should inform patient expectations and guide physician treatment practice.

Second, we found that active disease at the time of conception or during pregnancy was a predictor for postpartum flare. Disease activity has been a well-studied predictor of adverse pregnancy outcomes including preterm birth and low birth rate; conversely, studies have shown associations with preconception disease activity and IBD disease course during pregnancy. ${ }^{12,18}$ A recent meta-analysis of $>1700$ patients showed that patients with active disease at the time of conception were twice as likely to experience active disease during pregnancy when compared with patients in remission at conception. ${ }^{23}$ Similarly, a cohort study by Bortoli et al. showed that $26 \%$ of patients with active disease remained active throughout pregnancy, whereas only $14 \%$ of those in remission relapsed during pregnancy. ${ }^{24} \mathrm{To}$ expand upon these observations, our study demonstrates that active disease during conception and pregnancy subsequently impacts the risk of postpartum flare during the year after delivery. Of particular note, in our cohort, 5 women had active disease at the beginning of pregnancy, all of whom experienced a flare during the postpartum year. Furthermore, only $38.4 \%$ of patients with postpartum flares also had active disease in the third trimester, whereas the remaining represented those who developed new-onset symptoms during the postpartum period. These associations may be attributed to overlying inflammatory and immune pathways of pregnancy and IBD, involving immunomodulation of T-cell activity and proinflammatory cytokines. ${ }^{14,25}$ Our findings emphasize the critical importance of maintaining remission at the time of conception, as it not only influences pregnancy outcomes but also correlates with IBD disease activity both throughout pregnancy and during the vulnerable postpartum period.

Another key finding from our cohort was the observation that de-escalation of maintenance therapies, including stopping or decreasing the dose or frequency, during pregnancy or the postpartum year was independently associated with postpartum IBD flare. To date, almost all of the current medical therapies for IBD (with the exception of methotrexate) have a favorable safety profile and may be continued during pregnancy and postpartum. ${ }^{26-30}$ There is variation between different societies in recommendations for continuation of biologic therapy during pregnancy. Consistent with our findings,

TABLE 2. Multivariable Analysis of Independent Predictors of Postpartum Flare in Women With Inflammatory Bowel Disease

\begin{tabular}{lcc}
\hline Characteristic & Multivariate Adjusted Odds Ratio & $95 \%$ Confidence Interval \\
\hline Age & 0.92 & $0.85-0.99$ \\
Active disease in the third trimester & 6.97 & $2.81-17.27$ \\
Therapy de-escalation during pregnancy & 3.00 & $1.03-8.68$ \\
Therapy de-escalation after delivery & 4.43 & $1.55-12.65$
\end{tabular}


TABLE 3. Predictors of Postpartum Relapse Among Women on Biologic Therapy

\begin{tabular}{lrr}
\hline Characteristic & Postpartum Flare (n = 26), No. (\%) & No Postpartum Flare (n = 37), No. (\%) \\
\hline Therapy de-escalation during pregnancy & $7(26.7)$ & $5(13.5)$ \\
Therapy de-escalation after delivery & $8(30.8)$ & $3(8.1)$ \\
Biologic held 1st trimester & $4(15.4)$ & $3(8.1)$ \\
Biologic held 3rd trimester & $13(50.0)$ & $15(40.5)$ \\
Timing of last biologic, mean (SD), wk before delivery & $10.5(13.7)$ & 0.18 \\
Active disease by trimester & & 0.04 \\
First & $4(15.4)$ & $0.4(10.1)$ \\
Second & $9(34.6)$ & $2(5.4)$ \\
Third & $11(42.3)$ & $2(5.4)$ \\
\hline
\end{tabular}

the American Gastroenterological Association (AGA) pregnancy care pathway recommends continuation of 5-ASA therapies, immunomodulators, or biologics throughout pregnancy and delivery, with consideration of biologic dosing adjustment (without medication interruption) near the time of delivery to achieve drug trough at the estimated delivery date. ${ }^{31}$ In contrast, consensus recommendations from the European Crohn's and Colitis Organization suggest that clinicians may consider stopping anti-tumor necrosis factor (anti-TNF) agents at 24-26 weeks in a subset of patients with sustained remission to limit placental transfer. ${ }^{32}$ Additionally, previous studies have demonstrated modest rates of self-reported levels of nonadherence during pregnancy, often due to fear of side effects to the fetus, a practice that has been postulated to contribute to disease flare. ${ }^{33-35}$ Similarly, we observed that therapy de-escalation in our cohort was primarily patient-directed. Our study further demonstrates that medication de-escalation (both dose/frequency decrease and cessation) during pregnancy increases the risk of flare during the postpartum year, independent of disease activity during pregnancy, suggesting that there are definite adverse consequences to such therapy changes. In an analysis of the subgroup of patients on biologic therapy, medication cessation or dose decrease postdelivery was associated with risk of relapse. Cessation of therapy after pregnancy may be due to concern about safety during breastfeeding and perceived benign course of disease postpartum. A growing body of literature has demonstrated that levels of biologics in the breast milk are minimal and are unlikely to be associated with any adverse effects in the neonate. ${ }^{26,27,36,37}$ Consequently, both the safety of the therapy and the risk of postpartum flare with biologic cessation or dose decrease after delivery should be emphasized to women, and therapy adherence encouraged.

Finally, there were multiple factors that were not associated with IBD flare postpartum in our cohort. Similar to prior studies, mode of childbirth was not a predictor of IBD flare during the postpartum year. ${ }^{21,38}$ Furthermore, a large proportion of our patients breastfed $(87.4 \%)$, and we did not note a correlation between breastfeeding and postpartum flare, supporting a previous study by Moffatt et al. ${ }^{16}$ Though a European cohort demonstrated that pregnant women with UC were at higher risk of relapse during the 6 months postpartum, our study did not show a difference in postpartum flare in terms of IBD type. ${ }^{24}$ The difference may be attributed to more aggressive UC treatment in our cohort, as $30.5 \%$ of the patients in our cohort were on biologics at the time of conception, whereas the majority of patients in the European cohort were on 5-ASA monotherapy at the time of conception and few $(<2 \%)$ were on biologics.

We readily acknowledge several potential limitations in our study. First, because the data source is a cohort of women from tertiary care IBD centers, these patients may have more severe disease, as evidenced by the higher frequency of women on biologics as compared with previous studies. Thus, results may not be generalizable to the whole IBD population. Second, because our study was a retrospective cohort study, the results could be influenced by missing information and incomplete documentation. Due to this, our findings may underestimate the rate of postpartum flares due to inadequate documentation of care both inside and outside our system. Given the small sample size of patients on biologics, caution should be exercised when interpreting the comparisons of this subgroup. Finally, we did not include nonpregnant patients as controls in our study, as our primary aims were to identify the frequency and predictors of relapse rather than comparison with external controls.

In conclusion, our findings indicate that a third of patients with IBD may experience a relapse during the postpartum year. The strongest predictors of this relapse were therapy de-escalation and active disease from the time of conception to the third trimester. Optimal disease control and adherence to medical therapy are important to ensure optimal outcomes for women during the postpartum period. Studies of pregnancy in women with IBD should be systematically extended to also include the postpartum year to inform clinical practice. 


\section{REFERENCES}

1. Kappelman MD, Rifas-Shiman SL, Kleinman K, et al. The prevalence and geographic distribution of Crohn's disease and ulcerative colitis in the United States. Clin Gastroenterol Hepatol. 2007;5:1424-1429.

2. Cosnes J, Gower-Rousseau C, Seksik P, Cortot A. Epidemiology and natural history of inflammatory bowel diseases. Gastroenterology. 2011;140:1785-1794.

3. Molodecky NA, Soon IS, Rabi DM, et al. Increasing incidence and prevalence of the inflammatory bowel diseases with time, based on systematic review. Gastroenterology. 2012;142:46-54.e42; quiz e30.

4. Selinger CP, Eaden J, Selby W, et al. Inflammatory bowel disease and pregnancy: lack of knowledge is associated with negative views. J Crohns Colitis. 2013;7:206-13.

5. Carbery I, Ghorayeb J, Madill A, Selinger CP. Pregnancy and inflammatory bowel disease: do we provide enough patient education? A British study of 1324 women. World J Gastroenterol. 2016;22:8219-8225.

6. Selinger CP, Leong RW, Lal S. Pregnancy related issues in inflammatory bowel disease: evidence base and patients' perspective. World J Gastroenterol. 2012;18:2600-2608

7. Kornfeld D, Cnattingius S, Ekbom A. Pregnancy outcomes in women with inflammatory bowel disease - a population-based cohort study. Am J Obstet Gynecol. 1997;177:942-946.

8. Mahadevan U, Sandborn WJ, Li DK, et al. Pregnancy outcomes in women with inflammatory bowel disease: a large community-based study from Northern California. Gastroenterology. 2007;133:1106-1112.

9. Nørgård B, Hundborg HH, Jacobsen BA, et al. Disease activity in pregnant women with Crohn's disease and birth outcomes: a regional Danish cohort study. Am J Gastroenterol. 2007;102:1947-1954.

10. Bröms G, Granath F, Linder M, et al. Birth outcomes in women with inflammatory bowel disease: effects of disease activity and drug exposure. Inflamm Bowel Dis. 2014;20:1091-1098.

11. O'Toole A, Nwanne $\mathrm{O}$, Tomlinson $\mathrm{T}$. Inflammatory bowel disease increases risk of adverse pregnancy outcomes: a meta-analysis. Dig Dis Sci. 2015;60:2750-2761.

12. Reddy D, Murphy SJ, Kane SV, et al. Relapses of inflammatory bowel disease during pregnancy: in-hospital management and birth outcomes. Am J Gastroenterol. 2008;103:1203-1209.

13. Hendrick V, Altshuler LL, Suri R. Hormonal changes in the postpartum and implications for postpartum depression. Psychosomatics. 1998;39:93-101.

14. Nasef NA, Ferguson LR. Inflammatory bowel disease and pregnancy: overlapping pathways. Transl Res. 2012;160:65-83

15. Kane $\mathrm{S}$, Lemieux $\mathrm{N}$. The role of breastfeeding in postpartum disease activity in women with inflammatory bowel disease. Am J Gastroenterol. 2005;100:102-105.

16. Moffatt DC, Ilnyckyj A, Bernstein CN. A population-based study of breastfeeding in inflammatory bowel disease: initiation, duration, and effect on disease in the postpartum period. Am J Gastroenterol. 2009;104:2517-2523.

17. Julsgaard M, Nørgaard M, Hvas CL, et al. Self-reported adherence to medical treatment, breastfeeding behaviour, and disease activity during the postpartum period in women with Crohn's disease. Scand J Gastroenterol. 2014;49:958-966.

18. Agret F, Cosnes J, Hassani Z, et al. Impact of pregnancy on the clinical activity of Crohn's disease. Aliment Pharmacol Ther. 2005;21:509-513.

19. Pedersen N, Bortoli A, Duricova D, et al; European Crohn-Colitis OrganisationECCO-Study Group of Epidemiology Committee-EpiCom. The course of inflammatory bowel disease during pregnancy and postpartum: a prospective European ECCO-EpiCom Study of 209 pregnant women. Aliment Pharmacol Ther. 2013;38:501-512.

20. Nalichowski R, Keogh D, Chueh HC, Murphy SN. Calculating the benefits of a Research Patient Data Repository. AMIA Annu Symp Proc. 2006:1044.
21. Cheng AG, Oxford EC, Sauk J, et al. Impact of mode of delivery on outcomes in patients with perianal Crohn's disease. Inflamm Bowel Dis. 2014;20:1391-1398.

22. de Silva PS, Nguyen DD, Sauk J, et al. Long-term outcome of a third anti-TNF monoclonal antibody after the failure of two prior anti-TNFs in inflammatory bowel disease. Aliment Pharmacol Ther. 2012;36:459-466.

23. Abhyankar A, Ham M, Moss AC. Meta-analysis: the impact of disease activity at conception on disease activity during pregnancy in patients with inflammatory bowel disease. Aliment Pharmacol Ther. 2013;38:460-466.

24. Bortoli A, Pedersen N, Duricova D, et al; European Crohn-Colitis Organisation (ECCO) Study Group of Epidemiologic Committee (EpiCom). Pregnancy outcome in inflammatory bowel disease: prospective European case-control ECCOEpiCom study, 2003-2006. Aliment Pharmacol Ther. 2011;34:724-734.

25. Christiaens I, Zaragoza DB, Guilbert L, et al. Inflammatory processes in preterm and term parturition. J Reprod Immunol. 2008;79:50-57.

26. Gisbert JP. Safety of immunomodulators and biologics for the treatment of in flammatory bowel disease during pregnancy and breast-feeding. Inflamm Bowel Dis. 2010;16:881-895.

27. Gisbert JP, Chaparro M. Safety of anti-TNF agents during pregnancy and breastfeeding in women with inflammatory bowel disease. Am J Gastroenterol. 2013;108:1426-1438.

28. Narula N, Al-Dabbagh R, Dhillon A, et al. Anti-TNF $\alpha$ therapies are safe during pregnancy in women with inflammatory bowel disease: a systematic review and meta-analysis. Inflamm Bowel Dis. 2014;20:1862-1869.

29. Rahimi R, Nikfar S, Rezaie A, Abdollahi M. Pregnancy outcome in women with inflammatory bowel disease following exposure to 5-aminosalicylic acid drugs: a meta-analysis. Reprod Toxicol. 2008;25:271-275.

30. Coelho J, Beaugerie L, Colombel JF, et al; CESAME Pregnancy Study Group (France). Pregnancy outcome in patients with inflammatory bowel disease treated with thiopurines: cohort from the CESAME study. Gut. 2011;60:198-203.

31. Mahadevan $\mathrm{U}$, Robinson $\mathrm{C}$, Bernasko $\mathrm{N}$, et al. Inflammatory bowel disease in pregnancy clinical care pathway: a report from the American Gastroenterological Association IBD Parenthood Project Working Group. Gastroenterology. 2019; 156:1508-1524.

32. van der Woude CJ, Ardizzone S, Bengtson MB, et al; European Crohn's and Colitis Organization. The second European evidenced-based consensus on reproduction and pregnancy in inflammatory bowel disease. $J$ Crohns Colitis. 2015;9:107-124

33. Mañosa M, Navarro-Llavat M, Marín L, et al. Fecundity, pregnancy outcomes, and breastfeeding in patients with inflammatory bowel disease: a large cohort survey. Scand J Gastroenterol. 2013;48:427-432.

34. Nielsen MJ, Nørgaard M, Holland-Fisher P, Christensen LA. Self-reported antenatal adherence to medical treatment among pregnant women with Crohn's disease. Aliment Pharmacol Ther. 2010;32:49-58.

35. Julsgaard M, Nørgaard M, Hvas CL, et al. Self-reported adherence to medical treatment prior to and during pregnancy among women with ulcerative colitis. Inflamm Bowel Dis. 2011;17:1573-1580.

36. Ben-Horin S, Yavzori M, Kopylov U, et al. Detection of infliximab in breast milk of nursing mothers with inflammatory bowel disease. J Crohns Colitis. 2011;5:555-558.

37. Matro R, Martin CF, Wolf D, et al. Exposure concentrations of infants breastfed by women receiving biologic therapies for inflammatory bowel diseases and effects of breastfeeding on infections and development. Gastroenterology. 2018; 155:696-704

38. Ananthakrishnan AN, Cheng A, Cagan A, et al Mode of childbirth and long-term outcomes in women with inflammatory bowel diseases. Dig Dis Sci. 2015;60:471-477. 